\title{
Mechanical Effects in PEM Fuel Cell: Application to Modeling of Assembly Procedure
}

\author{
S. Martemianov ${ }^{1}$, M. Gueguen ${ }^{2}$, J.C. Grandidier ${ }^{2}$ and D. Bograchev ${ }^{3}$ \\ ${ }^{1}$ Laboratoire d'Etudes Thermiques, LET UMR-CNRS 6608, \\ Université de Poitiers - ESIP et ENSMA, 40, avenue du Recteur Pineau 86022 Poitiers Cedex France \\ ${ }^{2}$ Laboratoire de Physique et Mécanique des Matériaux, LMPM UMR CNRS 6617, \\ ENSMA, Téléport 2, 1, Av. Clément Ader, BP 40109 - 86962 FUTUROSCOPE Cédéx, France \\ ${ }^{3}$ Frumkin Institute of Physical Chemistry and Electrochemistry RAN, Moscow 117071, Leninsky prospect 31, Russia \\ E-mail: serguei.martemianov@univ-poitiers.fr
}

\begin{abstract}
Mechanical effects can influence significantly electrical performance and life time of PEM fuel cells. A linear elasticplastic 2D model of fuel cell with hardening is used for modeling of assembly procedure of fuel cells. The model simulates mechanical behavior of the main components of real fuel cell (the membrane, the gas diffusion layers, the graphite plates, and the seal joints) and clamping elements (the steel plates, the bolts, the nuts). The stress and plastic deformation in MEA have been calculated using ABAQUS code. The results are presented on the local and the global scales with respect to the realistic clamping conditions. The first one corresponds to the single tooth/channel structure. The global scale deals with features of the entire cell and takes into account the border effects, in particular the influence of seal joints.
\end{abstract}

Keywords: Fuel cell design, Proton exchange membrane (PEM), Nafion, ABAQUS.

\section{NOMENCLATURE}

$$
\begin{array}{cl}
\mathrm{d} \lambda & \text { the plastic multiplier } \\
\mathrm{E} & \text { the Young's modulus } \\
\delta_{\mathrm{ij}} & \text { the Kroneker symbols } \\
\varepsilon_{\mathrm{ij}}^{\mathrm{EL}} & \text { the elastic strain tensor }
\end{array}
$$

\section{INTRODUCTION}

Proton exchange membrane fuel cell (PEMFC) is a promising substitute of combustion engine owing to its zero level of pollutions and high efficiency. Nevertheless, the durability of fuel cells necessitates significant improvements (Chalk 2006). For automotive applications the required lifetime of PEMFC exceeds 5000h (Mathias 2005). The PEMFC lifetime limitations can be caused by chemical factors, for example, degradation of catalyst layers. Another reason deals with the thermomechanical deformation of Membrane Electrode Assembly (MEA) which is the heart of PEMFC. Today Nafion ${ }^{\circledR}$ is used in fabrication of MEA as a material for membrane. This polymer has excellent thermal and mechanical stability which allows the membrane to operate in chemical hostile environment with changes in temperature and humidity and under mechanical stresses.

Mechanical stresses arising in MEA have two origins. At the first, the initial stresses arise in the MEA during cell assemble procedure. Many efforts have been paid for optimization of this procedure (bolt assembling), in

$$
\begin{array}{cl}
\varepsilon_{\mathrm{ij}}^{\mathrm{PL}} & \text { the plastic strain tensor } \\
\sigma_{\mathrm{ij}} & \text { the stress tensor } \\
v & \text { the Poisson's ratio }
\end{array}
$$

particular for homogenisation of the arising mechanical stresses. The bolts assure the tightness of the fuel cell and the electrical conductivity between different contact elements. The fuel cell tests show that the contact pressure is one of the parameters which determine the performance of fuel cells (Kadjo 2006, 2007). Additional mechanical stresses arise during fuel cell running, because PEMFC consists of the materials with different thermal expansion and swelling coefficients. These additional mechanical stresses can be one of the sources of the MEA lifetime limitations.

In order to improve the durability of fuel cells, it is necessary to understand the phenomena which govern the arising of pinholes and delamination between membrane and gas diffusion layer (Kundu 2006). In particular, it is necessary to separate the mechanical, the thermal and the chemical effects in this problem. One of the ways in this direction deals with understanding of mechanical deformations arising in fuel cell.

Some studies have been provided for modeling of mechanical stresses in fuel cells. In the papers 
(Tang 2006, Kusoglu 2006, 2007) a 2D model has been developed and used for numerical simulations of mechanical stresses in MEA. Calculations have been performed for the single tooth/channel configuration. Thus, the obtained results reflect the local effects on the scale of only one channel and cannot be used for the prediction of the edging effects near the seal joints. Moreover, the classical boundary conditions (fixed displacement or fixed force) used in the previous works do not reflect reality of the fuel cell assembling. Indeed, the bolts act as flexible elements which impose the different stress depending on their elongation and their stiffness.

The aim of the present study is to fill up some gaps of the previous works. Stresses arising in a single fuel cell during the assembling are studied numerically by means of ABAQUS code. The calculations have been provided for a fuel cell geometry which is similar to the one used in the papers (Tang 2006, Kusoglu 2006, 2007). Nevertheless, significant improvements have been brought in the model. In order to take into account border effects, a single tooth/channel configuration of the previous works has been generalized and the mechanical stresses have been calculated in the entire fuel cell. Thus, all the channels and the joints are taken into account in the present model. The stresses arising during cell assembling are discussed on the scale of one channel and on the scale of the entire cell. The physical model of fuel cell, the numerical procedure and all parameters necessary for mechanical stresses calculation are presented with respect to the previous works.

\section{MODEL DESCRIPTION}

\subsection{Geometry}

The real three-dimensional geometry of fuel cell is presented on Fig. 1a. Because of numerical difficulties we use a reduced geometry in the present study and provide $2 \mathrm{D}$ modeling.
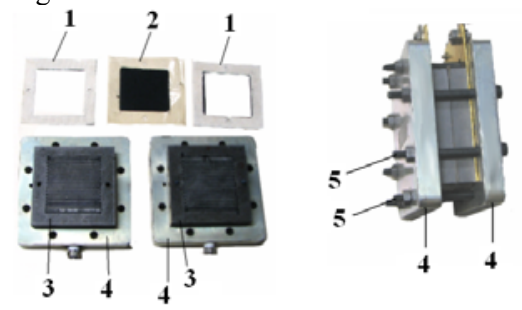

(a)

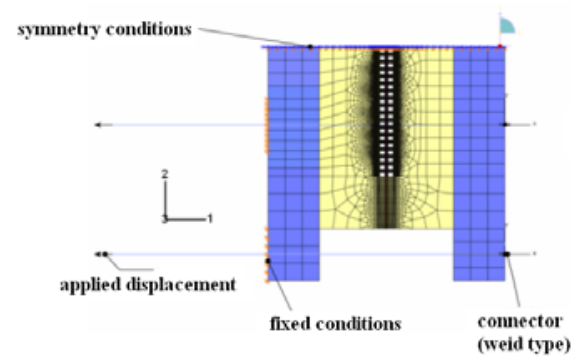

(b)

Fig. 1. a) Fuel cell components and fuel cell after assembly. b) Models mesh and boundary conditions.

1 - Seal joints, 2 - MEA, 3 - Graphite plates, 4 - Steel plates, 5 - Bolts.

Nevertheless, the present approach is more realistic in comparison with the previous studies (Tang 2006,
Kusoglu 2006, 2007), since the entire cross-section of a single fuel cell is considered (see Fig. 1b, Fig. 2). The used model consists of 8 main parts: two steel plates, two graphite plates, two gas diffusion layers (GDL), a proton exchange membrane and a seal joint. In addition, there are 2 fastening elements (bolts) allowing a realistic simulation of applied loading. The catalytic layers are thinner than the membrane by one order of magnitude, thus it is possible to neglect their influence on mechanical phenomena, i.e. the catalytic layers are integrated into the gas diffusion layer. The similar assumption was used in (Tang 2006, Kusoglu 2006, 2007).

The size of the problem has been reduced using a symmetry condition, in other words, we suppose that there is the axe of symmetry for all physical quantities (see Fig. 1b). The detailed diagram of a half cell is shown in Fig. 2.

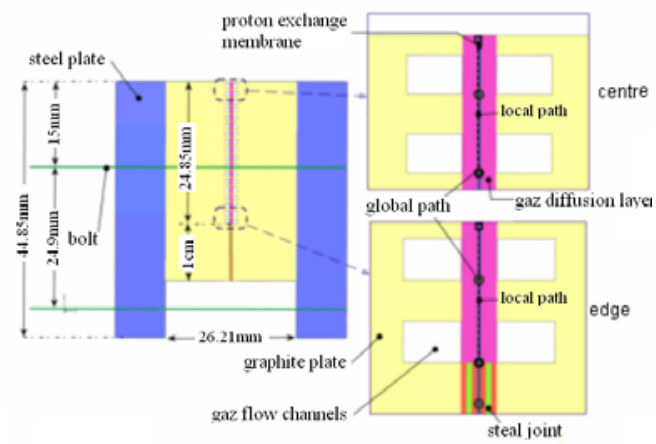

Fig. 2. a) Detailed schema of fuel cell model with zoomed edge and centre region

During the modeling the following parameters have been used: the thickness of the membrane $-0.05 \mathrm{~mm}$, the thickness of the steel plates $-10 \mathrm{~mm}$, the thickness of the GDE - $0.28 \mathrm{~mm}$, the length of the bolt $-80 \mathrm{~mm}$, the diameter of the bolt $-5 \mathrm{~mm}$ and the thickness of the seal joint $-0.3 \mathrm{~mm}$, the width of the tooth $-0.7 \mathrm{~mm}$ and the width of the channel $-0.7 \mathrm{~mm}$, the depth of channel $1 \mathrm{~mm}$. The dimension which is perpendicular to a plane of cross-section (2D model) equals $100 \mathrm{~mm}$. The other sizes are defined in the figure. The numerical model has a periodic pattern with the opposite channels. We suppose a perfect assembling of different elements: the sliding is not taken into account and it is accepted that the stresses are transmitted completely on the interfaces. This hypothesis leads to an overestimation of stresses in the plane of the membrane.

\subsection{Mechanical Properties}

In this model it is assumed that the steel, the carbon paper and the graphite plate have linear-elastic behavior. The mechanical properties of nafion membrane and carbon paper have been taken from Tang (2006).

Mainly, the present model follows the one described in the works (Tang 2006, Kusoglu 2006, 2007) but the seal joints are introduced in this study as an additional element. Proprieties of the seal joint have been associated with the one of Taconic $\AA$, which is composed by three layers of identical thickness: woven fiberglass coated with PTFE (polytetrafluoroethylene). Three layers of standard 
elements are used for modeling of seal joint, each layer is assumed to have elastic mechanical properties of fiberglass and PTFE.

Classical elasto-plastic model with isotropic hardening is accepted for Nafion ${ }^{\circledR}$ membrane. In reality, the membrane has viscoelastic behaviour. As a consequence, the present model ignores the effect of relaxation of stresses, the phenomenon which is intensified with the increase of the temperature. In this paper we study a cold assembling procedure; nevertheless it is important to note the restrictive character of the elasto-plastic model with respect to stresses. In our opinion the results obtained in the frame of this model give the overestimated values of stresses.

The main governing equations of the elasto-plastic model are specified below. The total strain tensor components are the sum of the elastic strain tensor components $\varepsilon_{\mathrm{ij}}^{\mathrm{EL}}$ and the plastic strain tensor components $\varepsilon_{\mathrm{ij}}{ }^{\mathrm{PL}}$ :

$\varepsilon_{i j}=\varepsilon_{i j}^{E L}+\varepsilon_{i j}^{P L}$.

These strains are assumed to be small; this hypothesis is not very restrictive as the materials are confined within the assemblage. The material behaviors are assumed to be isotropic, and in the elastic region the Hooke's law is accepted. The constitutive law can be written as follows:

$$
\sigma_{\mathrm{ij}}=\frac{\mathrm{E}}{(1+v)(1-2 v)}\left(v \varepsilon_{\mathrm{ij}}^{\mathrm{EL}}+\sum_{\mathrm{k}}(1-2 v) \varepsilon_{\mathrm{kk}}^{\mathrm{EL}} \delta_{\mathrm{ij}}\right),
$$

where $\sigma_{i j}$ are the stress tensor components, $v$ is the Poisson's ratio, E is the Young's modulus,. $\delta_{i j}$ is the Kroneker $\delta$-symbol.

The plasticity behavior is described by Prandtl-Reuss theory. The Von Mises yield function is written as follows:

$$
\mathrm{f}\left(\sigma_{\mathrm{ij}}\right)=\sqrt{\frac{3}{2} \mathrm{~S}_{\mathrm{ij}} \mathrm{S}_{\mathrm{ij}}}-\sigma_{0}
$$

and the Von Mises yield criterion is reduced to:

$$
\mathrm{f}\left(\sigma_{\mathrm{ij}}\right)=0 \text {, }
$$

where $\mathrm{S}_{\mathrm{ij}}=\sigma_{\mathrm{ij}}-\frac{1}{3} \sigma_{\mathrm{kk}} \delta_{\mathrm{ij}}$ is the deviatoric stress tensor components. The plasticity starts when $\mathrm{f}\left(\sigma_{\mathrm{ij}}\right)<0$. Plasticity evolutions are described by isotropic hardening curves of Nafion ${ }^{\circledR}$ which are taken from work the (Tang 2006). According to the Prandtl-Reuss theory, the plastic increment tensor is proportional to the derivative of the yield function $\mathrm{f}$, see Eq. (3):

$$
\mathrm{d} \varepsilon_{\mathrm{ij}}=-\frac{\partial \mathrm{f}}{\partial \sigma_{\mathrm{ij}}} \mathrm{d} \lambda \text {, }
$$

where $\mathrm{d} \lambda$ the plastic multiplier is calculated by the consistent relation $(\mathrm{df}=0)$ during the hardening. The plastic multiplier corresponds to the increment of equivalent plastic strain.

\subsection{Mechanical Loading}

In the previous works (Tang 2006, Kusoglu 2006, 2007), the mechanical loading of fuel cell has been considered using two schematizations: relative displacement of two plates and application of a given force. Both schematizations are traditional, but do not reflect the reality.

A more realistic approach is used in the present study, namely the bolts are introduced in the model. The bolts are modelled by the beams with circular section (type B21 in $A B A Q U S(R)$, which are attached to one of the plates using the connector join (type CONN2D2 in ABAQUS ${ }^{\circledR}$ ). The connection between the 2D mesh and the beam is realized by means of the total elimination of the degree of freedom in order to assume the perfect transmission of the forces and the moments from the head of the bolts to the plate. On the opposite plate, in the place where the nuts press the surface, the displacement in the normal direction is forbidden (see Fig. 1b). The loading is applied by a displacement of the second edge of the beam; this displacement reflects the loading arising during application of a moment of forces to the bolt. The displacement is imposed gradually and the contact pressure between the elements of the fuel cell is governed by the stiffness of the bolts. The size and the elasticity modulus of the bolts in the model have been chosen with respect to the ones used in the real assembling procedure. It is assumed that the assembly is provided at the room temperature $27^{\circ} \mathrm{C}$ and the moisture content of the Nafion ${ }^{\circledR}$ membrane equals $35 \%$.

In the real $3 \mathrm{D}$ configuration the bolts are disposed around the entire cell with aim to apply homogeneous contact pressure between Nafion ${ }^{\circledR}$ membrane, GDL and graphite plates. Only two loading beams are used in this study. However, we have verified afterwards that 2D model allows rather good simulating of homogeneous pressure distribution for the case of two parallelepiped plates joined by two loading beams.

In the real assembling procedure the operator uses a bolt torque as a reference experimental value. The reduction from the real $3 \mathrm{D}$ configuration to $2 \mathrm{D}$ model requires estimation of the normal stresses provided by bolts in numerical schema for a given bolt torque. It can be done by adjusting of the displacement applied to the edges of the both beams in numerical calculations to the one generated in the real fuel cell. In other words, a normal stress between graphite plate and steel plate in numerical calculations should be close to the mean pressure arising in a real fuel cell. This pressure allows establishing of a simple relation between displacements of beams in numerical model and bolts torque applied during cell assembly procedure.

It has been calculated that the applied displacement $\mathrm{d}_{\mathrm{IMP}}=0.038 \mathrm{~mm}$ allows obtaining of the average value $1 \mathrm{MPa}$ for the normal stress on the interface between graphite and steel plates. This value of loading has been accepted in (Tang 2006, Kusoglu 2006, 2007) and is used as the reference in this work. The corresponding pressing force applied to the beams equals $\mathrm{F}=1865 \mathrm{~N}$.

Using classical relation, it is possible to calculate the tightening torque $\mathrm{C}$ as a function of $\mathrm{F}$ - the tension applied to the screw and $\mu$ - the friction of the thread of the bolt:

$$
\mathrm{C}=\mathrm{F}\left(\mu \cdot \mathrm{D}_{\mathrm{ma}}+\mu \cdot \mathrm{D}_{\mathrm{mf}}+\mathrm{D}_{\mathrm{mf}} \cdot \tan (\alpha)\right) / 2,
$$


where $D_{m a}$ is the average diameter of the contact zone between the head and the steel plate, $D_{m f}$ is the diameter of the thread and $\alpha$ is the angle of the thread pitch. Supposing that the friction coefficient $\mu$ is approximately equal to 0.2 (dry assembling) and using the characteristics of standard screw (type M6 80), it is possible to obtain that the contact pressure $1 \mathrm{MPa}$ corresponds to experimental tightening torque $\mathrm{C}$ equal to $2.32 \mathrm{Nm}$.

\section{RESULTS AND DISCUSSION}

\subsection{Presentation of Results}

The developed model allows obtaining the distribution of the stresses in the membrane on two scales. The local scale corresponds to a single tooth/channel configuration; this local analysis has been performed in the cell center region (near the axis of symmetry) and in the cell edge region. The corresponding zones are identified on Fig. 2 (see the small dots in the zoomed pictures).

A particular global path is used for presentation of the stresses distribution on the scale of the entire cell. This path goes through the membrane center points which are situated in front of the lowest corners of the channels (see Fig. 2, the big dots in the zoomed pictures). The path also goes under the joint which gathers around the membrane in order to visualize the concentration of the stresses in this transitional zone. The normal direction 1 is perpendicular to the MEA and the transverse directions 2 and 3 are situated in the plane of the MEA; the direction 3 is perpendicular to the plane of the model (see Fig. 2).

\subsection{Global Distribution}

The stresses distributions on the scale of the entire fuel cell are presented in Fig. 3.

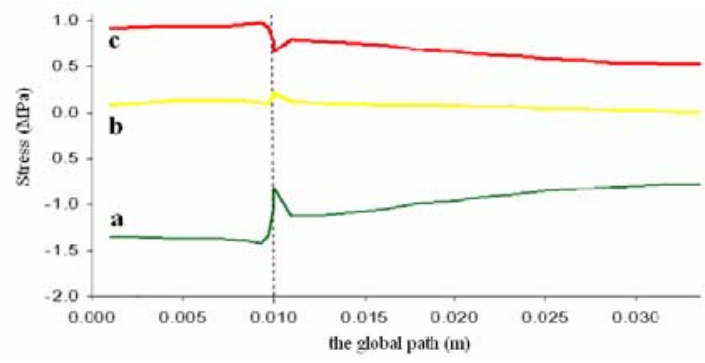

Fig. 3. a - Normal Stress, b - Shear stress, c - Misses stress.

A strong discontinuity in the stress distribution in the membrane under the interface between seal joint and GDL can be noted. This inhomogeneous distribution affects all the components of the stress tensor. In the membrane the most important component of the stress is the normal one, $\sigma_{11}$ (in the direction 1). It can be noted that $\sigma_{11}$ is negative along the membrane surface; this corresponds to the state of the compression. So, in this configuration the joint assumes its function. The transversal stresses are negative because the model ignores sliding between the elements of assembly. Naturally, the transversal stresses can decrease when the sliding occurs. The shear stresses are by one order of the magnitude smaller. For these stresses a peak can be observed under the joint/GDL interface. Near the cell centre the stresses decrease slightly with respect to the values obtained near the joints. The average value of the Mises stresses is about $0.72 \mathrm{MPa}$ in the central part of the cell. Under the seal joint, the compressive load in the membrane is higher than $1 \mathrm{MPa}$ (mean value imposed by bolts). However, under the diffusion layer the compressive stress is lower than mean value.

Numerical modeling of the arising stresses confirms some experimental results. In particular, it is known that the junction is the zone sensible to failure. The strong discontinuity observed numerically near the joint confirms this experimental fact. Non-uniformity of the stresses during assembly is caused by the structural features and non-uniform displacement (see Fig. 3) is generated by assembling elements (bolts). Pressure is higher in the cell edge than in the cell centre due to bending of the steel plates under the action of loading bolts. The difference between stiffness of seal joint (composite PTFE/woven fiberglass) and stiffness of gas diffusion layer (carbon paper) is the principal factor responsible for the singularity of the compressive stress at joint/GDL interface.

\subsection{Local Distribution}

The numerical simulations confirm that the stresses in the membrane have periodic evolution in phase with the periodicity of the channels. This result is obtained for all components of the stress tensor. The maximum amplitudes of stress oscillations are localized at the edge of the fuel cell and they are about of $1 \mathrm{Mpa}$ for the normal stress $\sigma_{11}$ and 0.30Mpa for tangential stress (see Fig. 4). Normal and tangential stress distributions have a similar character. The difference of the local values at a given position for each channel allows to quantify the global evolution on the channel scale (values at $\mathrm{x}=0 \mathrm{~m}$ and $\mathrm{x}=0.0015 \mathrm{~m}$ ).

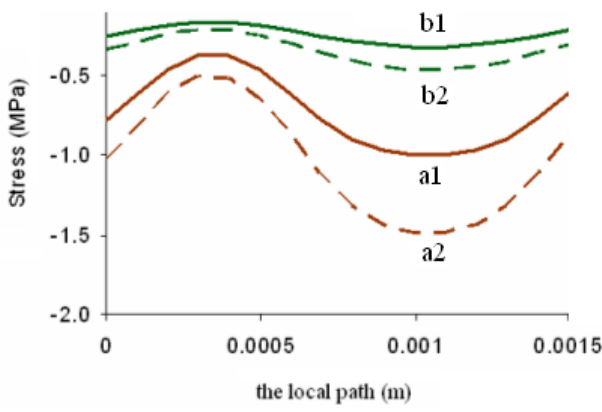

Fig. 4. Normal (a) and tangential (b) stresses distributions in the centre (1) and at the edge (2) region of the membrane.

The variations in global scale are small in comparison to the local oscillation of the stress field. From the local point of view, the stress state is strongly inhomogeneous. However, it should be marked that the tangential stresses are relatively important on the border of the cell and are higher in comparison to the ones in the centre. At the local scale the normal stresses are more important than the tangential and the shear ones, this point confirms the results observed at the global scale.

\subsection{On the Plasticity Effects}

It is noteworthy that in our model the plastic strain is not reached when the applied pressure is equal to $1 \mathrm{MPa}$ as well as in the articles (Tang 2006, Kusoglu 2006, 2007) under the identical conditions. The calculations have been 
performed in order to determine the torque which can cause the plastic effect in the membrane due to mechanical load; the results are presented on Fig. 5.

Taking the loading which generates normal stress of $1 \mathrm{MPa}$ as a reference point $(100 \%)$, the stresses distributions have been calculated for more important loading. It was shown that plasticity effects arise for loading which corresponds to 15.8 Nm (curve $\mathbf{c}$ in Fig. 5); this reflects the displacement of $0.258 \mathrm{~mm}$ imposed to the tightened screw. In this case the plasticity arises in the membrane under the seal joint (junction joint/ diffusion plate), the level of the equivalent plastic strain is nearly equal to 0.001 . The value of pressure in the graphite plate is about $7.3 \mathrm{MPa}$. The plastic zone expands under the seal joint and the level of equivalent plastic strain rises with the increase of compression (see Fig. 5) The curve b corresponds to the displacement of $0.266 \mathrm{~mm}$ with tightening torque which is equal to $16.3 \mathrm{Nm}$, and the curve a corresponds to a displacement of $30.410^{-}$ ${ }^{2} \mathrm{~mm}$ with tightening torque which is equal to $18.6 \mathrm{Nm}$.

The influence of the torque on the fuel cell performance has been studied experimentally in (Kadjo 2006, 2007). The maximal performance in these studies has been obtained with the compression moment of about $9 \mathrm{Nm}$. The cell assembly in this work has been provided by the screw bolts of M6 80 type. Taking into account the number of the bolts and the surface of the MEA, it is possible to estimate the pressure arising in the graphite plate as $4.1 \mathrm{MPa}$. These estimations show that the applied mechanical loading in real assembly procedure can be considerably larger than $1 \mathrm{MPa}$, the value accepted in (Tang 2006, Kusoglu 2006, 2007) for the numerical simulations. That is why the risk of the plastic effect has been underestimated in previous works.

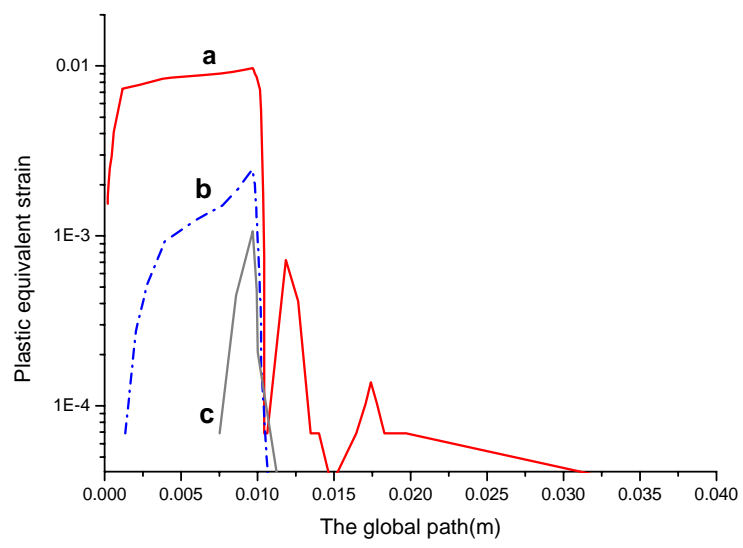

Fig. 5. Equivalent plastic strain for different initial loading: $800 \%$ - a; $700 \%$ - b; $680 \%$ - c; loading which generates the normal stress of $1 \mathrm{MPa}$ corresponds to $100 \%$.

\section{Conclusion}

2D finite elements model representing the entire fuel cell has been used for studies of mechanical stresses generated in MEA during assembly. The model assumes linear elastic behavior for the cell components except the membrane which has elasto-plastic behavior with hardening. The realistic assembly procedure is simulated taking into account mechanical action of flexible bolts. The simulations allow understanding of the stress distributions on the local (single tooth/channel structure) and the global (entire fuel cell) scales. On the both scales the important spatial variations of stresses generated during the assembling procedure have been detected; this inhomogeneity can limit mechanical reliability of the fuel cell system. In particular, a zone with strong heterogeneous stresses in membrane under the junction seal joint/graphite plate has been observed and quantified. This heterogeneity is caused mainly by the structural features and by the difference between stiffness of seal joint and gas diffusion layer. Calculations show that the membrane reaches plasticity under the torque which is equal $15.8 \mathrm{Nm}$. The plasticity arises primarily in the zone of the maximal stresses, i.e. under the junction seal joint/graphite plate. This effect has not been identified in previous works which have been performed for rather low mechanical loading. In consequence, for improvement of lifetime characteristics of fuel cells it is necessary to take carefully into account the arising of mechanical stresses in MEA.

\section{ACKNOWLEDGMENT}

The authors thank the federation of the laboratories of Poitiers P'PRIMME and the CNRS program PICS $\mathrm{N}^{\circ}$ 4323 for the financial support of this work.

\section{REFERENCES}

Chalk, S.J. and J.F. Miller (2006). Key challenges and recent progress in batteries, fuel cells, and hydrogen storage for clean energy systems. Journal of Power Sources 159(1), 73-83.

Mathias, M.F., R. Makharia, H.A. Gasteiger, J.J. Conley, T.J. Fuller, C.J. Gittleman, S.S. Kocha, D.P. Miller, C.K. Mittelsteadt, T. Xie, S.G. Yan and P.T. Yu (2005). Two Fuel Cell Cars in Every Garage? Electrochemical Society Interface 14(3), 24-35.

Kadjo, J.J., P. Brault, A. Caillard, C. Coutanceau, J.P. Garnier and S. Martemianov (2007). Improvement of proton exchange membrane fuel cell electrical performance by optimization of operating parameters and electrodes preparation. Journal of Power Sources 172(2), 613-622.

Kadjo, J.J., J.P. Garnier, J.P. Maye, F.S. Relot and S. Martemianov (2006). Performance and instabilities of proton exchange membrane fuel cells. Russian Journal of Electrochemistry 42(5), 467-475.

Kundu, S., M.W. Fowler, L.C. Simon and S. Grot (2006). Morphological features (defects) in fuel cell membrane electrode assemblies. Journal of Power Sources 157(2), 650-656.

Kusoglu, A., A.M. Karlsson, M.H. Santare, S. Cleghorn and W.B. Johnson (2006). Mechanical response of fuel cell membranes subjected to a hygro-thermal cycle. Journal of Power Sources 161(2), 987-996.

Kusoglu, A., A.M. Karlsson, M.H. Santare, S. Cleghorn and B.J. William (2007). Mechanical behavior of fuel cell membranes under humidity cycles and effect of swelling anisotropy on the fatigue stresses. Journal of Power Sources 170(2), 345-358.

Tang, Y., A.M. Karlsson, M.H. Santare, M. Gilbert, S. Cleghorn and W.B. Johnson (2006). An experimental 
S. Martemianov et. al / JAFM, Vol. 2, No. 2, pp. 49-54, 2009.

investigation of humidity and temperature effects on the mechanical properties of perfluorosulfonic acid membrane. Journal of Material Science and Engeniring 425(1-2), 297-304.

Tang, Y., M.H. Santare, A.M. Karlsson, S. Cleghorn and W.B. Johnson (2006). Stresses in proton exchange membranes due to hydro-thermal loading. Journal of Fuel Cell Science and Technology 3(2), 119-124. 